\title{
Bacteriological Profile and Antibiogram of Bacterial Isolates from Pus Samples in Tertiary Care Hospital of Kathmandu
}

\author{
Upendra Pandeya ${ }^{1^{*}}$, Mithileshwor Raut ${ }^{2}$, Saru Bhattarai ${ }^{3}$, Padam Raj Bhatt ${ }^{1}$, Puspa Raj Dahal ${ }^{1}$ \\ ${ }^{1}$ Department of Microbiology, Tri-Chandra Multiple Campus, Kathmandu, Nepal \\ ${ }^{2}$ Department of Biochemistry TUTH, Kathmandu, Nepal \\ ${ }^{3}$ Department of Microbiology, GoldenGate International College, Kathmandu, Nepal
}

*Corresponding author: Upendra Pandeya; Department of Microbiology, Tri-Chandra Multiple

Campus, Kathmandu, Nepal; Email: pandeyaupendra@gmail.com

\begin{abstract}
Objectives: The main aim of the study was to isolate and identify the bacterial agent and to determine the susceptibility pattern of isolates to different antibiotics.

Methods: Thisretrospective study was conducted from February to October 2015 in microbiology laboratoryof All Nepal Hospital Kathmandu, Nepal. The clinical specimens were processed for isolation and identification of bacteriafollowing standard microbiological procedures. Antibiotic susceptibility pattern of isolates were determined according to CLSI guidelines (CLSI 2014)

Results: A total of 271 clinical specimens were processed where 164 (60.5\%) showed growth positivity. A total 164 bacterial isolates were detected among which 84 (51.22\%) were Gram positive $80(48.78 \%)$ were Gram negative bacteria. Thirteen different species of bacteria were isolated. The most prevalent isolate was Staphylococcus aureus 53 (32.30\%) followed by E. coli 34 (20.80\%), (CoNS) 15 (9.10\%), Klebsiella pneumoniae 15 (9.10\%), Enterococcus fecalis 12(7.30\%), Pseudomonas aeruginosa $10(6.10 \%)$, Acinetobacter spp. 7 (4.30\%) Citrobacter spp., Proteus spp., Klebsiella oxytoca were less common. S. aureus was most susceptible to Amikacin.Vancomycin was the most effective drugs for Enterococcus fecalis. Among Gram negative bacteria E. coli was found most sensitive to Polymyxin B (100\%) and Imipenem (76.5\%) where Pseudomonas aeruginosa was sensitive to, Amikacin, Imipenem (80\%). Polymyxin B was the most effective drugs for Klebsiella pneumoniae. Acinetobacter spp. was found highly resistant to different antibiotics.
\end{abstract}

Conclusion: Antibiotic susceptibility evaluation showed Aminoglycosides, Phenicols Polymyxin, and Imipenem was the most effective drugs overall.

Key words: Wound infection, Staphylococcus aureus, Antibiogram, Aminoglycosides

\section{INTRODUCTION}

Skin, the largest organ in the human body, plays a crucial role in the sustenance of life through regulation of water and electrolyte balance, thermoregulation, and by acting as a barrier to external noxious agents including microorganisms (Zafar et al. 2008). Agents that causes wound infection can be classified on the basis of depth of wound and likelihood that they serve as the carrier for organisms that cause infection (Shrestha 2009).

There are three major sources of wound contaminantsexogenous sources (i.e. water-borne from water related injury or microorganisms from soil in a soilcontaminated injury or air-borne), endogenous source (i.e. microorganisms colonizing sweat glands, hair follicles or mucosa of gastro intestine, oropharynx, genitourinary tract) and the surrounding skin (File and Tan 1995; Acharya et al. 2008). Presence of pathogenic bacteria in wound doesn't imply infection. Infection occurs when one or more of the contaminants evades the clearing effect of the host's defenses, replicates in large numbers, attacks and harms the host's tissues (Colle et al. 1996). Wound infection is a major problem in Nepal. A complication of wound infection is very common because of poor hospital management and poor aseptic techniques used in hospitals during surgical procedure or other hospital procedure. 


\section{MATERIALS AND METHODS}

A retrospective study was conducted February to October 2015 in the laboratory of All Nepal Hospital in order to find out the causative agent of wound infection and their antibiotic susceptibility pattern. The population for this study was both inpatient (admitted to different wards) and outpatient who had been requested for culture and antibiotic susceptibility from suspected wound infections by the medical practitioners or by physicians Macroscopic examination was carried out to note the colour, consistency and the presence of granules. All wound swab specimens were inoculated on Blood Agar (BA) plate, MacConkey Agar (MA) and nutrient agar (NA) and incubated at $37^{\circ}$ for 18-24 hours. (Benson 2001; Cheesbrough 2006) Preliminary identification of bacterial isolates were done bypigmentation, haemolysis on BA and also by Gram staining Conventional biochemical tests were performed from primary cultures for identification of the isolates. Gram negative rods were identified by performing a series of biochemical tests namely: catalase test, oxidase test methyl-red (MR) test, Voges-Prouskaure (VP) test indole test, motility ,hydrogen sulphide $\left(\mathrm{H}_{2} \mathrm{~S}\right)$ production test, triple sugar iron (TSI), Citrate utilization and urease test. Gram positive cocci were identified by catalase test, oxidase test, OF test and coagulase test (Benson 2001; Cheesbrough 2006).
Antibiotic Susceptibility Testing (AST): The antibiotic susceptibility of isolates were determined by Kirby- Bauer disc diffusion method using Clinical and Laboratory Standard Institute (CLSI) guidelines (2014). Antibiotics used were Amoxicillin, Amikacin, Azithromycin, Cefixime,,Cefotaxime, Cefoxitin, Ceftazidime, Chloramphenicol, Colistin, Co-Trimoxazole, Doxycycline, Gentamicin, Imipenem, Levofloxacin, Nalidxilic acid, Nitrofurontoin, Norfloxacin, Ofloxacin, Piperacillin/ Tazobactam, Polymyxin B and Tigecycline. Those isolates which were non susceptible (either a resistant or intermediate) to three or more antibiotic classes were regarded as MDR (Magiorakos et al. 2011).

\section{RESULTS}

Out of 271 samples studied 153 (56.5\%) were pus swab and $118(43.5 \%)$ were aspirated pus in which $164(60.5 \%)$ samples showed bacterial growth while 107 (39.5\%) samples showed no growth. Among 164 positive samples, $89(54.3 \%)$ were aspirated pus and 75 (45.7\%) were pus swab that shown growth positive.

\section{Distribution of Bacterial agents}

Among 164 growth positive sample most prevalent bacteria was $S$. aureus $(32.3 \%)$ which was followed by E. coli $(20.7 \%)$. The least isolated bacteria was Proteus mirabils $(0.6 \%)$.

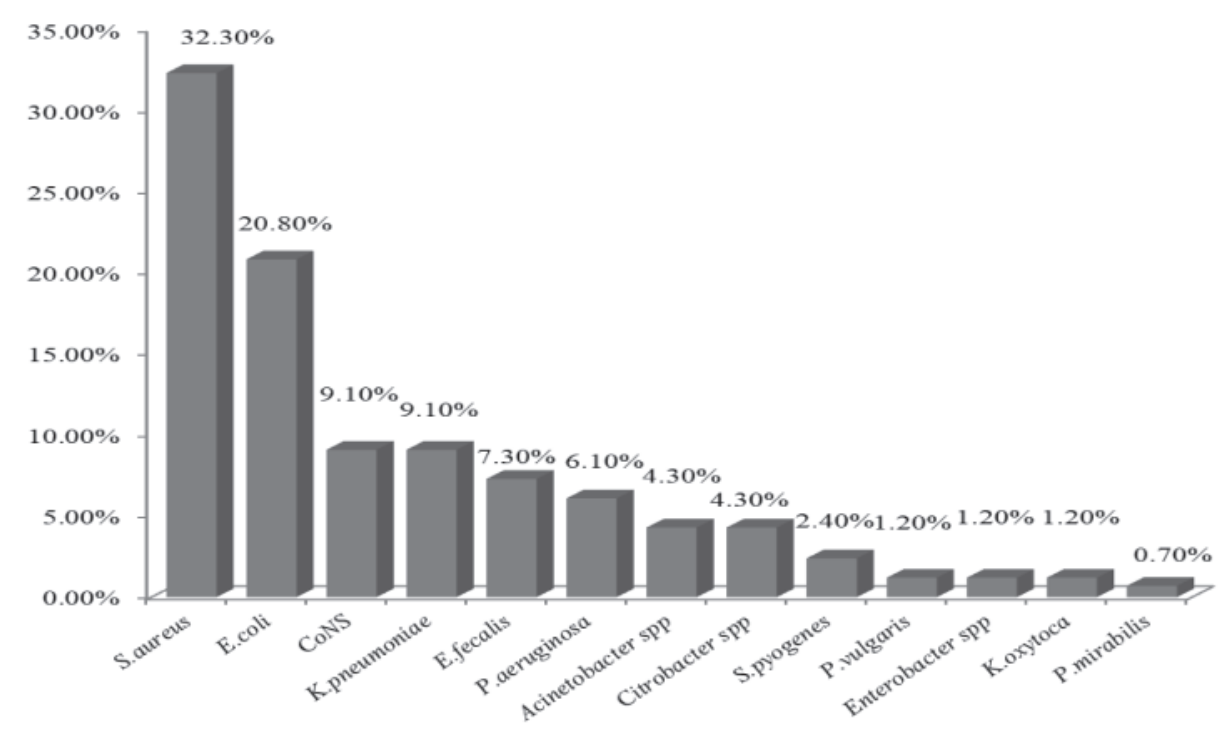

Figure 1: Percentage distribution of total bacterial isolates from wound 
Antibiotic Susceptibility Pattern of the Bacterial Isolates Antibiotic susceptibility pattern of E. coli: Among different antibiotics used the most effective antibiotic was Polymyxin B which was 100\% sensitive followed by Imipenem (76.5\%) and Amikacin (70.6\%). The least effective antibiotic was Ampicillin (100\%) resistant followed by Cefepime and Ceftazidime were (88.2\%) resistant.

Table 1: Antibiotic susceptibility pattern of E. coli

\begin{tabular}{|c|c|c|c|c|c|c|c|}
\hline \multirow{2}{*}{ Antibiotics } & \multicolumn{2}{|c|}{ Sensitive } & \multicolumn{2}{|c|}{ Intermediate } & \multicolumn{2}{|c|}{ Resistant } & \multirow{2}{*}{ Total } \\
\hline & No & $\%$ & No & $\%$ & No & $\%$ & \\
\hline Ampicillin & - & - & - & - & 34 & 100 & 34 \\
\hline Cotrimoxazole & 9 & 26.5 & - & - & 25 & 73.5 & 34 \\
\hline Ciprofloxacin & 6 & 17.6 & 3 & 8.8 & 25 & 73.5 & 34 \\
\hline Chloramphenicol & 19 & 55.9 & 2 & 5.9 & 13 & 38.2 & 34 \\
\hline Imipenem & 26 & 76.5 & 2 & 5.9 & 6 & 17.6 & 34 \\
\hline Amikacin & 24 & 70.6 & - & - & 10 & 29.4 & 34 \\
\hline Gentamicin & 23 & 67.6 & - & - & 11 & 32.4 & 34 \\
\hline Cefepime & 4 & 11.8 & - & - & 30 & 88.2 & 34 \\
\hline Ceftazidime & 4 & 11.8 & - & - & 30 & 88.2 & 34 \\
\hline Cefotaxime & 10 & 29.4 & - & - & 24 & 70.6 & 34 \\
\hline Tetracycline & 5 & 14.7 & 2 & 5.9 & 27 & 79.4 & 34 \\
\hline Polymyxin B & 34 & 100 & - & - & - & - & 34 \\
\hline Piperacillin/Tazobactam & 17 & 50 & 9 & 26.5 & 8 & 23.5 & 34 \\
\hline
\end{tabular}

Antibiotic susceptibility pattern of Pseudomonas aeruginosa: The most effective antibiotic for the Pseudomonas aeruginosa was Amikacin, Chloramphenicol, and Imipenem having the sensitivity

Table 2: Antibiotic susceptibility pattern of $P$. aeruginosa

\begin{tabular}{|c|c|c|c|c|c|c|c|}
\hline Antibiotics & \multicolumn{2}{|c|}{ Sensitive } & \multicolumn{2}{|c|}{ Intermediate } & \multicolumn{2}{|c|}{ Resistant } & Total \\
\hline Ampicillin & - & - & - & - & 10 & 100 & 10 \\
\hline Gentamicin & 7 & 70 & - & - & 3 & 30 & 10 \\
\hline Amikacin & 8 & 80 & - & - & 2 & 20 & 10 \\
\hline Ciprofloxacin & 5 & 50 & 2 & 20 & 3 & 30 & 10 \\
\hline Chloramphenicol & 8 & 80 & - & - & 2 & 20 & 10 \\
\hline Imipenem & 8 & 80 & - & - & 2 & 20 & 10 \\
\hline Tetracycline & 2 & 20 & - & - & 8 & 80 & 10 \\
\hline
\end{tabular}

Among different antibiotics used the most effective antibiotic was Polymyxin B (100\%) and followed by of $80 \%$ followed by Gentamycin and Piperacillin/ Tazobactam of $70 \%$ sensitivity. The least sensitive antibiotic was Tetracycline (20\%).
Piperacillin/Tazobactam (73.2\%). The least effective antibiotic was Ampicillin (100\%) and Ceftazidime (86.7\%). 
Pandeya et al. 2017; TUJM 4(1): 55-62

Table 3: Antibiotic susceptibility pattern of Klebsiella pneumoniae

\begin{tabular}{|c|c|c|c|c|c|c|}
\hline \multirow{3}{*}{ Antibiotics used } & \multicolumn{6}{|c|}{ Susceptibility pattern } \\
\hline & \multicolumn{2}{|c|}{ Sensitive } & \multicolumn{2}{|c|}{ Intermediate } & \multicolumn{2}{|c|}{ Resistant } \\
\hline & No. & $\%$ & No. & $\%$ & No. & $\%$ \\
\hline Ampicillin & - & - & - & - & 15 & 100 \\
\hline Gentamicin & 6 & 40 & - & - & 9 & 60 \\
\hline Amikacin & 8 & 53.3 & 1 & 6.7 & 6 & 40 \\
\hline Cefepime & 5 & 33.3 & - & - & 10 & 66.7 \\
\hline Cefotaxime & 4 & 26.7 & - & - & 11 & 73.3 \\
\hline Ciprofloxacin & 4 & 26.7 & - & - & 11 & 73.3 \\
\hline Imepenem & 8 & 53.3 & - & - & 7 & 46.7 \\
\hline Ceftazidime & 2 & 13.3 & - & - & 13 & 86.7 \\
\hline Chloramphenicol & 6 & 40 & - & - & 9 & 60 \\
\hline Cotrimoxazole & 5 & 33.3 & - & - & 10 & 66.7 \\
\hline Piperacillin/Tazobactam & 11 & 73.3 & 2 & 13.3 & 2 & 13.3 \\
\hline Polymyxin B & 15 & 100 & - & - & - & - \\
\hline Tetracycline & 6 & 40 & - & - & 9 & 60 \\
\hline
\end{tabular}

The most effective drug against $S$. aureus was Amikacin (94\%) followed by Gentamycin (92.5\%) and Chloramphenicol (77.4\%). Among different antibiotics used least effective was Penicillin (96.2\%). CoNS were highly sensitive towards Amikacin and Chloramphenicol (80\% both) and Gentamicin (66.7\%).

Table 4: Comparative antibiotic susceptibility pattern of S. aureus and CoNS

\begin{tabular}{lcccccc}
\hline \multirow{2}{*}{ Antibiotic } & \multicolumn{3}{c}{ S.aureus $(\%)(\mathrm{N}=53)$} & \multicolumn{3}{c}{ CONS (\%) $(\mathrm{N}=\mathbf{1 5})$} \\
\cline { 2 - 7 } & $\mathrm{S}$ & $\mathrm{I}$ & $\mathbf{R}$ & $\mathrm{S}$ & $\mathrm{I}$ & $\mathbf{R}$ \\
\hline Penicillin & 3.8 & - & 96.2 & 13.3 & - & 86.7 \\
Erythromycin & 37.3 & 9.4 & 52.8 & 20 & 13.3 & 66.7 \\
Cotrimoxazole & 32.1 & 7.5 & 60.4 & 33.3 & 13.3 & 53.3 \\
Tetracycline & 52.8 & - & 47.2 & 53.3 & - & 46.7 \\
Chloramphenicol & 77.4 & - & 22.6 & 80 & - & 20 \\
Ciprofloxacin & 39.6 & 9.4 & 50.9 & 46.7 & - & 53.3 \\
Gentamicin & 92.5 & 1.9 & 5.7 & 66.7 & - & 33.3 \\
Amikacin & 94.3 & 1.9 & 3.8 & 80 & - & 20 \\
\hline
\end{tabular}

The most effective antibiotic for E. fecalis was Tetracycline, Chloramphenicol and Vancomycin (100\%) whereas least effective antibiotic was Ciprofloxacin $(58.3 \%)$.

Table 5: Antibiotic susceptibility pattern of Enterococcus fecalis

\begin{tabular}{lccccccc}
\hline \multirow{2}{*}{ Antibiotics } & \multicolumn{2}{c}{ Sensitive } & \multicolumn{2}{c}{ Intermediate } & \multicolumn{2}{c}{ Resistant } & \multirow{2}{*}{ Total } \\
\cline { 2 - 6 } & No & $\%$ & No & $\%$ & No & $\%$ & 12 \\
\hline Ampicillin & 8 & 66.7 & - & - & 4 & 33.3 & 12 \\
Erythromycin & 6 & 50 & 2 & 16.7 & 4 & 33.3 & 12 \\
Tetracycline & 12 & 100 & & - & - & - & 12 \\
Chloramphenicol & 12 & 100 & - & - & - & - & 12 \\
Ciprofloxacin & 5 & 41.7 & - & - & 7 & 58.3 & 12 \\
Gentamicin & 8 & 66.7 & - & - & 4 & 33.3 & 12 \\
Vancomycin & 12 & 100 & - & - & - & - & 12 \\
\hline
\end{tabular}




\section{DISCUSSION}

Wound infection has been major concern among health care practitioners not only in terms of increased trauma but also in view of its burden on financial resources and the increasing requirements for cost effective management within health care system. Infection of wound delays in healing and may cause herniation of the wound and complete wound dehiscence (Alexender 1994). Wound infections are also significant in that they are the most common nosocomial infection (Dongi et al. 20011)

The study was designed with an aim to assess the prevalence of wound infection in All Nepal Hospital; the effect of age, gender and other co- morbid conditions in the prevalence as well as identifying the etiological agents and their susceptibility to antimicrobial agents. In this study a total 271 pus samples were collected and processed. The etiological agents were identified by culture and different biochemical tests and their susceptibility pattern with commonly used antibiotics were determined.

In our study, out of total samples from patients with wound infection, $60.5 \%$ showed bacterial growth whereas $39.5 \%$ didn't show any growth. Culture negative results might be difficulty in growing of fastidious organisms. Another possibility could be manual error in collection, transport of culture media and diagnosis of the infection itself and most probable reason was the sample from patient taking antibiotic. Similar studies conducted by KC et al. 2013 (60.2\%), Acharya et al. 2008 (50.7\%) and Bhatt et al. 2007 (80.6\%) findings in Nepal. Neelima et al. (2013) $58 \%$ of the sample cultured aerobically showed positive growth. A study conducted by Giacometti et al. (2000), Manyahi (2012) in tertiary hospital $>90 \%$ shows growth. Both of these studies were contrary to this study. This difference in prevalence may be due to variation in common nosocomial pathogens inhabitant, difference in policy of infection control and prevention between countries and hospitals and study designed used in the researches.

Out of total cases $46.9 \%$ were male patients where $53.1 \%$ female patients. The growth was found higher in male patients $53 \%$ and female $47 \%$.In this study, on total sample analyzed 139 (51\%) samples from outpatients and $132(49 \%)$ samples from inpatients while on growth positive 164 cases 88 (53.7\%) from inpatients and 76
(46.3\%) from outpatients shown microbial growth.

On total growth positive pus samples, 84(51.22\%) were Gram positive bacteria. Among Gram positive bacteria, S. aureus (63.1\%) was the most common isolates similar study conducted by Pokhrel et al. (2004) 57.66\%, Bhatt and Lakhey (2007) 50\%, Acharya et al. (2008) 51.2\%, showed $S$. aureus was the predominant in wound infection study conducted in Nepal, Mishra et al. (2000) reported 60.1\%. But Banjara et al. 2003 (24.9\%) from TUTH and De et al. 2003 (11.2\%) from India that showed lower frequency of S. aureus. CoNS (17.9\%) constituted second most prevalent bacteria among Gram positive bacteria in our study. Similar study carried by Neelima et al. (2013) CoNS (18.6\%) was the second predominant organisms. According to Shah et al. (1997) CoNS was $11.4 \%$ and Manyahi et al. (2012) it was the second most predominant but Altoparlak et al. 2004 reorted $63 \%$ of prevalence of CoNS which is contradictory with our findings. Similarly Enterococcus fecalis $(14.3 \%)$ and Streptococcus pyogenes (4.8\%) was found in our study. Yah et al. (2007) found that $S$. pyogenes $(3.3 \%)$ on kerosene burn wound.

In this study, antibiotic susceptibility test was performed for all bacterial isolates. The antibiotic discs used were Ciprofloxacin, Cotrimoxazole, Chloramphenicol, Amikacin, Gentamicin and Tetracycline was common antibiotics while Ampicillin was used for all Gram negative and Enterococcus fecalis. Penicillin and Erythromycin were only used for Gram positive isolates. Vancomycin was used only for Enterococcus fecalis. Antibiotics Ceftazidime Cefepime, Cefotaxime, Imipenem, Piperacillin/Tazobactam and Polymyxin B were used only for Gram negative. Cefoxitin disc was only used for S. aureus.

In our study, the most effective antibiotic for E. coli was Polymyxin B (100\%), Imipenem (76.5\%), Amikacin (70.6\%), Gentamycin (67.6\%) and Chloramphenicol (55.9\%). Other antibiotics like Ampicillin (100\%), Ceftazidime (88.2\%), Cefepime (88.2\%) and Tetracycline (79.4\%) resistance to E. coli. Yakha et al. (2014) showed Imipenem (96.4\%), Amikacin (86.6\%) and Piperacillin/ Tazo (70.7\%), Rao et al. (2014) reported Imipenem, Amikacin and Pipera/Tazo were (80\%) sensitive while Ampicillin (53.34\%), Ceftriaxone (73.34\%), Ciprofloxacin (73.34\%) show higher resistance to Ecoli. E. coli showed (90.9\%) resistant to Ampicillin and Ciprofloxacin, $(81.8 \%)$ to Cefotaxim and Ceftriaxone, 
(72.7\%) resistant to Cotrimoxazole. However, it was highly sensitive to Amikacin (100\%) followed by Gentamicin (54.5\%).

Regarding the sensitivity pattern of Pseudomonas spp., it was found that Amikacin, Imipenem, Chloramphenicol was the most effective drug $(80 \%)$ sensitivity and Gentamycin and Piperacillin/ Tazobactam showed 70\% sensitivity while Ampicillin, Cefepime and Tetracycline was the least effective drugs. Similarly a study carried by Amatya et al. (2015) reported Imipenem (87.9\%) and Amikacin (64.6\%) sensitive similarly Acharya et al. (2008) reported Amikacin was the most effective drug against $P$. aeruginosa. Pseudomonas aeruginosa was sensitive to Gentamicin (87.5\%) and Ceftazidime (85.7\%) but showed resistance to Ciprofloxacin (57.2\%) (Anguzu and Ohila 2007). Mengesha et al. (2014) found that $P$. aeruginosa were $100 \%$ resistant to Ceftriaxone, Amoxicillin, Tetracycline and Ampicillin. Similar resistant pattern was also shown by Guta et al. (2014).

The most effective antibiotic against Klebsiella pneumoniae was Polymyxin B (100\%). Antibiotics like Piperacillin/Tazobactam (73.2\%), Amikacin (53.3\%), Imipenem (53.3\%) sensitive to K. pneumoniae. The least effective antibiotics are Ampicillin (100\%), Ceftazidime (86.7\%), Cefotaxime and Chloramphenicol (73.3\%). Cotrimoxazole, Gentamicin Cefepime Tetracycline shows resistant above (60\%). Klebsiella oxytoca is highly susceptible to Polymyxin B and Amikacin (100\% both) other antibiotics like Imipenem, Cefotaxime, Chloramphenicol Tetracycline Shown (50\%) sensitive by K. oxytoca. A study conducted by Chowdhury et al. (2013) reported Klebsiella are highly sensitive to Imipenem (100\%) and Gentamicin Ceftazidime and Ceftriaxone are highly resistant to Klebsiella spp. Similarly Rao et al. (2014) have similar result with the present study, which shows maximum sensivity to Imipenem, Amikacin and Piperacillin/Tazobactam (76.92\%) of each, but higher resistant to Ciprofloxacin, Ampicillin and Cefotaxime.

In the present study, isolates of Acinetobacter spp. was found highly resistant to commonly used antibiotics Ampicillin, Ceftazidime, Cefotaxime (100\%) and Tetracycline (71.4\%), Amikacin (57.1\%), Gentamycin (71.4\%). Whereas Imeipenem (57.1\%) sensitive. Manyahi (2012) reported that Acinetobacter spp. were highly resistant to Ceftazidime, Ciprofloxcin and Gentamicin and $40 \%$ of them being resistant to Carbapenams. Idomir et al. (2009) also reported all tested antibiotics are resistant by Acinetobacter spp. except Carbapenam.

For Citrobacter spp. Amikacin and Polymyxin B (85.7\%) was the most effective antibiotic and Imipenem and Pipera/Tazo (71.4\%) and highly resistant to Ampicillin $(85.7 \%)$, Cefepime (100\%), Cotrimoxazole (71.4\%). Proteus spp are highly susceptible to Imipenem, Chlormphenicol, Ceftazidime, Amikacin, Cefepime and Gentamicin (100\%) but resistant to Polymyxin B, Ampicillin and Cotrimoxazole (100\%).

Resistance to Penicillins and Cephalosporins (Levy and Marshall 2004) by Gram negative bacteria is most commonly due to the production of $\beta$ - lactamase, either chromosomally encoded or, more often, plasmid mediated. Other important mechanisms of resistance include alteration in penicillin binding protein (PBPs), decreased penetration of the antibiotics to the bacterial cell or combinations of these resistance strategies (Deloney and Schiller 2000). Active efflux pumps in Gram negative bacteria which execrete drugs including multidrug efflux pumps, can also confer to resistance to $\beta$-lactams

The most effective antibiotic against $S$. aureus was Amikacin (94.3\%) followed by Gentamicin (92.5\%) and Chloramphenicol (77.4\%), only Cefoxitin (56.6\%). Antibiotics such as Erythromycin, Cotrimoxazle and Ciprofloxacin have sensitivity less than $40 \%$ and least effective antibiotic was Penicillin (96.2\%). The result was similar in case of CoNS being most sensitive with Chioramphenicol (80\%), Amikacin (80\%), followed by Gentamicin (66.7\%), Tertacycline (53.3\%) and Cefoxitine (53.3\%) sensitive.

The least effective antibiotic was Penicillin (86.7\%), Erythromycin and Cotrimoxazole. A study carried out by Amatya et al. (2015) in B \& B Hospital Nepal, Chloramphenicol (89.1\%) and Gentamicin (52.2\%) which is similar to our findings. Poudel (2013) reported that Chloramphenicol (98.9\%) and Gentamicin (86.8\%) was the most effective antibiotics against $S$. aureus which agreed with our findings. Andhoga et al. (2002) in Kenya have reports $S$. aureus being highly resistance to Chloramphenicol (84.8\%). S. aureus causes clinically relevant infections mostly because of its virulence factors such as coagulase, catalase clumping factor A and leucocidines (Dissemond 2009). 


\section{CONCLUSION}

In this study Gram positive bacteria was found predominant over Gram negative bacteria. The most common isolates were S. aureus (32.3\%), E. coli $(20.7 \%)$, K. pneumoniae $(9.1 \%)$ and P. aeruginosa (6.1\%). Pattern of bacterial isolates were similar in both inpatient and outpatients. The least effective antibiotic was Ampicillin (100\% resistant) in case of Gram negative and Penicillin (100\% resistant) in case of Gram positive. Acinetobacter spp. was highly resistant to different antibiotics. Polymyxin B was the most effective antibiotic against Gram negative bacteria like E. coli, Klebsiella spp., Proteus spp. and Citrobacter spp. Antibiotics like Amikacin, Gentamicin and Chloramphenicol were highly effective to Gram positive bacteria.

\section{ACKNOWLEDGMENTS}

Authors would like to thank all the patients who agreed to participate in this study and made this research possible. Furthermore extend our gratitude to All Nepal Hospital for providing the necessary laboratory facility.

\section{REFERENCES}

Acharya J, Mishra SK, Kattel HP, Rijal BP and Pokhrel BM (2008) Bacteriology of wound infection among patients attending TU Teaching Hospital, Kathmandu Nepal. JNAMLS 9: 76-80

Alexender MF (1994) Wound infection. in.; nursing practice hospital and home, Margaret F. Alexender, Josephine N. Fawcett, Phyllis J. Rucimang (eds). Churchill Livingstone, New York. Pg. 703.

Altoparlak U, Erol S, Akcay MN, Celebi F and Kadanali A (2004) The time related changes of antimicrobial resistance patterns and predominant bacterial profiles of burn wounds and body flora of burned patients. 30: 660-664.

Amatya J, Rijal M and Baidya R (2015) Bacteriological study of the postoperative wound samples and antibiotic susceptibility pattern of the isolates in B \& B Hospital. JSM Microbiology 3:1019.

Andhoga J, Macharia AG, Maikuma IR, Wanyonyi ZS, Ayumba BR and Kakai R (2002) Aerobic pathogenic bacteria in post- operatie wounds at MOi Teaching and Referral Hospital. East Afr Med J 79: 640-644.

Anguzu JR and Ohila D (2007) Drug sensitivity pattern of bacterial isolates from septic Post-operative wounds in a regional referral Hospital in Uganda. Africian Journal of Health Science 7:14854

Banjara MR, Sharma AP, Joshi AB, Tuladhar NR, Ghimire P and Bhatta DR (2003) Surgical wound infections in patients of TU teaching. J Nepal Health Res Council 1(2): 41-45.

Benson T (2001) Microbiological applications laboratory manual in general Microbiology. 8th Edition, The McGraw-Hill, New York.

Bhatt CP and Lakhey M (2007) The distribution of pathogens causing wound infection and their antibiotic susceptibility pattern. J Nepal Health Res Council 5: 22-26.

Cheesbrough M (2006) District laboratory practice in tropical countries part 2. 2nd edition. Cambridge University Press, New York.

Chowdhury D, Jhora ST, Shah MR and Nahar N (2013) Antibacterial resistance pattern of common bacterial pathogen in tertiary care hospitals in Dhaka City. Bangladesh J Med Microbiol 07: 13-16.

CLSI, Performance Standards for Antimicrobial Susceptibility Testing; Twenty-Fourth Informational Supplement. M100-S24, January 2014, 34: 1 .

Collee JG, Duguid JP, Fraser AG, Marmion BP and Simmons A (1996) Laboratory strategy in the diagnosis of infective syndromes. Mackie and McCartney practical medial microbiology; $14^{\text {th }}$ edition. Churchill Livingstone pp 53-94.

De A, Varaiya A, Mathur M and Bhesania A (2003) Bacteriological studies of gas gangrene and related infections. Indian J Med Microbiol 21: 202204.

Deloney CR and Schiller NL (2000) Characterization of an in vitro selected amoxicillin-resistant strain of Helicobacter pylori. Antimicrob Agents Chemother 44: 3368-3373

Dissemond J (2009) Methicillin resistant Staphylococcus aureus (MRSA): diagnostic, clinical relevance and therapy. J Dtsch Dermatol Ges 6: 544-551.

Dongi R, Rovera F, Dionigi G, Imperatori A, Ferrari A, Dionigi $O$ and Dominioni I (2001) Risk factors in Surgery. Journal of Chemotherapy 13: 6-11. 
File TM and Tan JS (1995) Treatment of skin and soft tissue infections. Am J Surg 169: 27-33.

Giacometti A, Cirioni O, Schimizzi AM, Del MS, Prete F, Barchiesi M.M.D, Errico E, Petrelli G and ScaliSe J (2000) Epidemiology and microbiology of surgical wound infection. J Clin Microbiol 38: 918-922.

Guta M, Aragaw K, and Merid Y (2014) Bacteria from infected surgical wounds and their antimicrobial resistance in Hawassa University Referral Teaching Hospital, Southern Ethiopia. AJMR 8: 1118-124.

Idomir M, Netmet C, Pascu A and Ardeleanu M (2009) Acinetobacter spp. pathogenic role and resistance to antibiotics. Bulletin of the Transilvania University of Brasov 2: 56-59.

KC R, Shrestha A and Sharma VK (2013) Bacteriological study of wound Infection and antibiotic susceptibility pattern of the isolates. Nepal Journal of Science and Technology 14: 143-150.

Levy SB and Marshall B (2004) Antibacterial resistance worldwide: causes, challenges and responces. Nature medicine supplement. 10: S122-S129.

Magiorakos AS, Srinivasan A, Carey RB, Carmeil Y, Falagus ME, Giske CG, Harbarth S, Hindler JF, Kahlmeter G, Olsson liljequist B, Paterson DL, Rise LB, Stelling J, Struelens MJ, Vatopoulos A, Weber JT and Monnet DL (2011) Multidrug resistant, extensively drug resistant and pandrug resistant bacteria: An international expert proposal for intense standard definition for acquired resistance. Clinical Microbiology and Infection ECDC 18: 268-281.

Manyahi J (2012) Bacteriological Spectrum of post-operative wound infections and their antibiogram in a tertiary hospital, Dares Salaam, Tanzania. A dissertation Submitted in Fulfillment of the Requirement for the Degree of Master of Medicine (Microbiology and Immunology) of Muhimbili University of Health and Allied Sciences Muhimbili University of Health and Allied Sciences.

Mishra RN, Chander Y, Debata NK and Ohri VC (2000)
Antibiotic resistance pattern of isolates from Wound and soft tissue infections. MJAFI 56

Neelima, Pravin DK, Suresh P and Nandeeshwar (2013) Bacteriological profile of wound infection in rural hospital in R.R district. Int J Med Res Health Sci 2: 469-473.

Pokhrel BM and Shrestha L (2004) Microbiology of burns wound in children. JNAMLS 6: 7-10.

Poudel P (2013) Bacteriological spectrum of wound infection and their antibiogram in a tertiary care hospital. A dissertation presented to the Department of Microbiology, Tri-Chandra Multiple Campus, Tribhuvan University, Kathmandu Nepal.

Shah PK, Pokhrel BM and Sharma AP (1997) The incidence of coagulase negative Staphylococci in Nepalese people. Nep Med Assoc 123: 296-300.

Rao R, Ranjan B and Debika RB (2014) Aerobic bacterial profile and antimicrobial susceptibility pattern of Pus isolates in a South Indian Tertiary Care Hospital. IORS-JDMS e-ISSN: 2279-0853, p-ISSN: 2279-0861. 13(3): 59-62

Shrestha P (2009) Study of bacteriological profile of infected wound from patients visiting Kanti children's hospital, Maharajgunj, Kathmandu. A M.Sc. dissertation presented to the Central Department of Microbiology, Tribhuvan University, Kathmandu, Nepal. pp 5-70.

Yah SC, Eghafona NO, Oranusi S and Abouo AM (2007) Wide spread plasmid resistance genes among Proteus spp. in diabetic wounds patients in ABUTH Zaria. Afr J Biotechnol 6: 1757-1762.

Yakha JK, Sharma AR, Dahal N, Lekhak B and Banjara MR (2014) Antibiotic susceptibility pattern of bacterial isolates causing wound infection among the patients visiting B \& B Hospital. Nepal Journal of Science and Technology 15: 91-96.

Zafar A, Anwar N and Ejaz H. (2008). Bacteriology of infected wounds- A study conducted at Children's Hospital Lahore. Biomedica 24: 71-74. 